\title{
Surface Gravity Waves: Shallow Water Fluid Particle Physics
}

\author{
Kern E. Kenyon \\ 4632 North Lane, Del Mar, CA, USA
}

Correspondence to: Kern E. Kenyon, kernken@aol.com

Keywords: Shallow Water Waves, Fluid Particle Physics

Received: September 12, $2020 \quad$ Accepted: October 24, $2020 \quad$ Published: October 27, 2020

Copyright $\odot 2020$ by author(s) and Scientific Research Publishing Inc.

This work is licensed under the Creative Commons Attribution International License (CC BY 4.0).

http://creativecommons.org/licenses/by/4.0/

(c) (i) Open Access

\section{ABSTRACT}

One of Newton's mathematical solutions to a hypothetical orbital problem, recently verified by an independent physics model, is applied to the fluid particle motion in shallow water surface gravity waves. What is the functional form of the central force, with origin at the ellipse's center, which will keep a body in the orbit? Newton found out it is the spring force, which is linear. All fluid particles in shallow water waves move in ellipses. By a superposition of solutions in a linear problem, the application of Newton's result to shallow water waves is combined with a feature not noticed by Newton: the orbital period is independent of the semi-major and semi-minor axes. Two conclusions reached are that the wave period of shoaling waves should be constant and that there is no friction in these waves.

\section{INTRODUCTION}

The present basically mathematical status of shallow water surface gravity waves, with respect to describing the paths of the fluid particles, is enhanced below by incorporating a physical model into the discussion.

According to laboratory studies that have used small neutrally buoyant particles in a wave tank, it is inferred that the motion of fluid particles in propagating surface gravity waves are in circles when the mean depth of water greatly exceeds the wavelength $[1,2]$. These are called deep water waves.

Shallow water waves occur when the waves travel in water with a mean depth comparable to or less than the wavelength. Classical wave theory predicts that the particle paths are ellipses when the waves are in shallow water. Whether or not the elliptical particle paths have been demonstrated in the lab is not known to me.

From the fluid dynamics text books $[3,4]$ comes the standard mathematical description of the elliptical particle paths, which is based on potential flow theory applied to surface gravity waves and that took a firm hold since the 1800 s. Something is missing here though which a question will bring out. During an orbital period, the total energy of each fluid particle evidently should remain constant, but does an ex- 
change between potential (PE) and kinetic (KE) energy take place? That would seem to be a reasonable thing going on during a wave period. For example, at the semi-major axes in the horizontal planes is probably where the maximum $\mathrm{PE}$ occurs and at the semi-minor axes in the vertical planes is where the maximum KE occurs.

However, the classical wave model is incapable of answering this question. Digging deeper is required which turns out to mean going further back in time to the 1600s, Newton's Principia in particular.

\section{METHOD}

It is now better known [5] that Newton warmed up to solving the famous and practical Kepler problem by working out four hypothetical exercises involving different functional forms for a central force acting on an orbiting body. During Newton's time, and for a long time after, the four hypothetical orbital problems he solved had no practical applications. One of the four hypothetical orbital examples is selected here for further study leading to an application: what form does the central force take to keep a body in an elliptical path when the force origin is at the center of the ellipse? Newton found by using a mathematical technique that the answer was the spring force. Recently Newton's method has been double checked by an independent physics model and verified for all five of his exercises [5].

Using the force balance method and plane polar coordinates $(r, \theta)$ the following equation of motion has been derived [5]

$$
\frac{1}{r}+\frac{\mathrm{d}^{2}}{\mathrm{~d} \theta^{2}} \frac{1}{r}=r^{2} \frac{f(r)}{m k}
$$

where $f(r)$ is the functional form of the central force, and $\mathrm{m}$ and $\mathrm{k}$ are constants. The two forces in the balance are the outward centrifugal force and the inward central force.

Also in polar coordinates the form for an elliptical orbit where the force center is at the center of the ellipse is

$$
\frac{1}{r^{2}}=\frac{\cos ^{2} \theta^{2}}{a^{2}}+\frac{\sin ^{2} \theta}{b^{2}}
$$

where $\mathrm{a}$ and $\mathrm{b}$ are constants (the semi-major and semi-minor axes). It can be verified that (2) is a solution of (1) when the spring force is put in for the central force function $f(r)$.

Now every fluid particle in a shallow water surface gravity wave has an elliptical orbit. Why not apply Newton's solution then to them all? Remember the central force, the spring force, is linear. Superposition of solutions applies to linear problems but not to nonlinear ones. Tradition has always regarded the surface gravity wave as being nonlinear, but here a breaking away from this point of view is occurring.

One feature of the elliptical problem apparently not noticed by Newton, or anybody else for that matter, is that the orbital period of the particle is independent of the parameters of the ellipse: the semi-major and minor axes as well as the orientation in the plane [6]. Also perturbing the particle in the plane of motion is predicted to not change the orbital period. That constant period characteristic is completely consistent with observations of the propagating shallow water surface gravity wave including the extreme case of the fluid particles at the flat solid bottom oscillating back and forth in straight lines. All particles have the same period independent of shape, of which there are an infinite number. And circular orbits of the deep water waves fall under the same analysis.

\section{DISCUSSION}

Above the question was raised about the exchange of KE and PE of a fluid particle during the elliptical orbital movement of a fluid particle in a shallow water surface gravity wave. With the exception of the circular orbit the answer is yes based on the physics involved.

In hindsight, in the early days of ships sailing the open seas, when obtaining longitudes was virtually impossible, it is conceivable that an accurate clock could have been made from a mass rotating on a spring 
in a plane. Perturbations acting on the orbiting particles are not expected to change the period [6].

\section{CONCLUSIONS}

Using the reasoning presented, the following prediction is offered. Considering that a shallow water surface gravity wave is propagating into very gradually decreasing mean depth of water, the wave period should remain constant during the shoaling process.

Another forecast due to the linearity of the problem is that ideally friction is zero in the shallow water surface gravity wave. This property was also advanced for deep water waves which helps explain how it is that swell can travel up to half way around the world according to observations [7].

\section{CONFLICTS OF INTEREST}

The author declares no conflicts of interest regarding the publication of this paper.

\section{REFERENCES}

1. Sommerfeld, A. (1964) Mechanics of Deformable Bodies. Academic Press, New York, 396 p.

2. Wiegel, R.L. (1964) Oceanographical Engineering. Prentice-Hall, New Jersey, 532 p.

3. Proudman, J. (1953) Dynamical Oceanography. John Wiley, New York, 377-380.

4. Kundu, P.K. (1990) Fluid Mechanics. Academic Press, San Diego, 190-200.

5. Kenyon, K.E. (1999) Newton's Hypothetical Orbits Independently Derived. Physics Essays, 39-43.

6. Kenyon, K.E. (2001) Exciting a Rotating Mass on a Spring without Change to its Rotation Rate. European Journal of Physics, 22, 471-475. https://doi.org/10.1088/0143-0807/22/5/301

7. Kenyon, K.E. (2020) Frictionless Surface Gravity Waves. Natural Science, 12, 199-201. https://doi.org/10.4236/ns.2020.124017 